\title{
Mixture prioritization and testing: the importance of toxicokinetics
}

\author{
Albert Braeuning $^{1}\left[\right.$ P Philip Marx-Stoelting ${ }^{2}$
}

Received: 2 March 2021 / Accepted: 9 March 2021 / Published online: 17 March 2021

(c) The Author(s) 2021

The topic of mixture toxicity has gained increased awareness in the past few years. Toxicological risk assessment is still mainly based on the analysis of individual compounds, while the consideration of mixture effects is already stipulated in some cases, e.g. in the field of pesticides. Given the almost infinite number of chemicals and their combinations, there is a need to develop strategies for grouping compounds and for the prioritization of potentially critical mixtures, which might exert effects that deviate from the general assumption of dose addition.

This may be achieved by approaches, which classify chemicals according to their toxicological properties, as, for example, established for pesticides: here, the active compounds are grouped into so-called common assessment groups (CAGs) (EFSA 2008, 2009, 2013). Even though these CAGs consider different aspects of hazard and do thus also consider some aspects of toxicokinetics, they set a strong focus on toxicodynamics. Common target organs or mechanisms of action, as they e.g. form the basis of the CAGs, are frequently used as a basis for the selection of chemicals for mixture testing. One example is the EUfunded project EuroMix: within that project, methodologies and tools for mixture risk assessment have been developed anchored in the adverse outcome pathway (AOP) concept and mixture selection mainly based on the toxicodynamic profiles of the test chemicals (Alarcan et al. 2021; Beronius et al. 2020).

Martin et al. (2021) have recently published a systematic review article on experimental analysis of chemical mixtures. The authors conclude that the dose addition concept appears to be suitable for most mixtures, while nonetheless

Albert Braeuning

albert.braeuning@bfr.bund.de

1 Department of Food Safety, German Federal Institute for Risk Assessment, Max-Dohrn-Str. 8-10, 10589 Berlin, Germany

2 Department of Pesticides Safety, German Federal Institute for Risk Assessment, Max-Dohrn-Str. 8-10, 10589 Berlin, Germany the potential of some classes of chemicals to produce nonadditive, especially synergistic mixture effects, should not be disregarded. Interestingly, the authors show that many published synergistic effects relate to metabolic, i.e. toxicokinetic interference. Of note, this is in accordance with observations from the pharmaceutical field demonstrating that pharmacokinetic interference is a major cause of undesired drug-drug interactions (Martin et al. 2021).

In line with the notion that toxicokinetic differences appear to play a relevant role as the underlying cause of nonadditive effects, a recent paper by Lasch et al. demonstrates that triglyceride accumulation in human liver cells caused by a triazole-class fungicide can be exacerbated by the presence of an additional, non-steatotic compound, and that this effect is related to an interference of the second compound with the cytochrome P450-mediated metabolism of the fungicide (Lasch et al. 2021). The findings presented in the paper highlight the importance of toxicokinetic interactions as a major cause of non-additive mixture effects. Moreover, the fact that the combination of a steatotic compound with a non-steatotic compound was able to amplify the observed cellular steatotic effect also highlights possible limitations of a mixture prioritization strategy solely relying on common toxicodynamic properties of the mixture constituents.

CAG- and AOP-based approaches are undoubtedly useful tools for grouping and mixture selection. Nonetheless, it appears that by focusing on toxicodynamic characteristics, the importance of toxicokinetic interferences as a mechanistic basis for non-additive mixture effects tends to be disregarded and underrated. Therefore, it appears timely that categorization of compounds, besides CAG grouping or similar strategies, should additionally be performed on a toxicokinetic basis, covering e.g. induction and inhibition of metabolic enzymes as well as interference related to effects at transport proteins. Therefore, it is proposed to develop a novel grouping approach based on common kinetic groups (CKG), to identify, select and prioritize compounds and their mixtures, which could interact at the toxicokinetic level leading to toxic effects beyond the assumption of additivity. 
Funding Open Access funding enabled and organized by Projekt DEAL.

\section{Compliance with Ethical Statement}

Conflict of interest The authors declare that there is no conflict of interest.

Open Access This article is licensed under a Creative Commons Attribution 4.0 International License, which permits use, sharing, adaptation, distribution and reproduction in any medium or format, as long as you give appropriate credit to the original author(s) and the source, provide a link to the Creative Commons licence, and indicate if changes were made. The images or other third party material in this article are included in the article's Creative Commons licence, unless indicated otherwise in a credit line to the material. If material is not included in the article's Creative Commons licence and your intended use is not permitted by statutory regulation or exceeds the permitted use, you will need to obtain permission directly from the copyright holder. To view a copy of this licence, visit http://creativecommons.org/licenses/by/4.0/.

\section{References}

Alarcan J, Sprenger H, Waizenegger J et al (2021) Transcriptomics analysis of hepatotoxicity induced by the pesticides imazalil, thiacloprid and clothianidin alone or in binary mixtures in a 28-day study in female Wistar rats. Arch Toxicol 95(3):1039-1053 https://doi.org/10.1007/s00204-020-02969-y
Beronius A, Zilliacus J, Hanberg A, Luijten M, van der Voet H, van Klaveren J (2020) Methodology for health risk assessment of combined exposures to multiple chemicals. Food Chem Toxicol 143:111520. https://doi.org/10.1016/j.fct.2020.111520

EFSA Panel on Plant Protection Products and their Residues (2008) Opinion of the scientific panel on plant protection products and their residues to evaluate the suitability of existing methodologies and if appropriate, the identification of new approaches to assess cumulative and synergistic risks from pesticides to human health with a view to set MRLs for those pesticides in the frame of regulation (EC) 396/2005. EFSA J 704:1-84

EFSA Panel on Plant Protection Products and their Residues (2009) Scientific opinion on risk assessment for a selected group of pesticides from the triazole group to test possible methodologies to assess cumulative effects from exposure through food from these pesticides on human health. EFSA J 7(9):1167

EFSA Panel on Plant Protection Products and their Residues (2013) Scientific opinion on the identification of pesticides to be included in cumulative assessment groups on the basis of their toxicological profile. EFSA J 11(7):3293

Lasch A, Marx-Stoelting P, Braeuning A, Lichtenstein D (2021) More than additive effects on liver triglyceride accumulation by combinations of steatotic and non-steatotic pesticides in HepaRG cells. Arch Toxicol. https://doi.org/10.1007/s00204-021-02997-2

Martin O, Scholze M, Ermler S et al (2021) Ten years of research on synergisms and antagonisms in chemical mixtures: a systematic review and quantitative reappraisal of mixture studies. Environ Int 146:106206. https://doi.org/10.1016/j.envint.2020.106206

Publisher's Note Springer Nature remains neutral with regard to jurisdictional claims in published maps and institutional affiliations. 